\title{
Future space challenges
}

\section{Oscar Adriani*}

Università degli Studi di Firenze and INFN Firenze

E-mail: adrianiefi.infn.it

\section{Paolo Papini}

INFN Firenze

\section{Piero Spillantini}

Università degli Studi di Firenze

\section{Elena Vannuccini}

\section{INFN Firenze}

In this paper a short review of the most challenging problems related to direct measurements of cosmic ray from space is presented. In particular a proposal on a possible future experiment to explore both the above- $\mathrm{TeV}$ energy region for antiparticles and the $\mathrm{PeV}$ region for nuclei is described. The experiment will adopt a large volume cylindrical shaped calorimeter, surrounded by a superconducting toroidal magnetic system, allowing precise measurements of the momentum of the charged cosmic ray up to few $\mathrm{TeV}$, and excellent particle identification and energy measurement up to the knee region.

INFN Workshop on Future Detectors for HL-LHC

16-18 December, 2015

Aula Magna della Cavallerizza Reale, Torino, Italy

\footnotetext{
*Speaker.
} 


\section{Introduction}

The study of cosmic radiation, which spans over a very wide energy range (up to $10^{20} \mathrm{eV}$, for charged particles), has made major progresses since the beginning of the space era. The possibility to operate particle detectors in space, without the natural shield provided by the atmosphere and by the magnetosphere, opened extremely interesting observational windows, giving the possibility to explore energy domains where ground-based instruments are blind and to investigate new physics phenomena.

Space experiments are very difficult and challenging, but also very exciting, since they allow to do direct measurements of cosmic rays in a very broad energy range, from the keV region (low energy $\mathrm{X}$-ray) up to the $\mathrm{PeV}$ region (high energy cosmic rays around the knee). The real breakthrough of the last two decades is connected to the possibility to use the advanced particle-detector techniques (developed for the standard ground based experiments, typically at the accelerators) in space, thanks to the improved technologies adopted in the electronic components design, that make them intrinsically more radiation hard, and to dedicated tests for the spatialisation of the detection systems. INFN played a crucial role in this process, being one of the leading institute in the very successful complex cosmic rays space experiments that have been launched in the last few years (AMS-01, Agile, Pamela, Fermi, AMS-02).

Several R\&D activities are currently in progress, facing very different technological issues for the next generation space experiments, that should overcome the limitation of the current experiments. A short overview of the most challenging ones is given in section 2 for the low energy region, while in section 3 a more detailed discussion is dedicated to charged cosmic rays in the $\mathrm{TeV}-\mathrm{PeV}$ energy region, where a novel concept design for a calorimeter combined with a magnetic spectrometer is presented.

\section{The low energy frontier}

The low energy region for both charged cosmic rays and photons can be investigated only with a space based experiment, since the atmospheric interactions prevent any type of measurements with a ground based experiment. The physics that can be investigated is very broad, spanning from the most intriguing astrophysical aspects in the $\mathrm{X}$-ray and $\gamma$ ray domain, to the dark matter investigation. In this section we will focus only on two items that are particularly interesting from the technological point of view.

\section{- X-ray polarimetry}

Spectroscopy, imaging and timing are routine techniques in the X-ray astronomy, that underwent continuous development over the last four decades. The possibility to measure the polarisation of the X-ray is potentially very useful to better understand many astrophysical phenomena, since a significant X-ray linear polarisation is expected in most classes of non-thermal X-ray sources, allowing to probe:

- emission processes in the sources (synchrotron radiation and inverse Compton, and acceleration phenomena in the supernova remnants, pulsar wind nebulae and jets); 
- geometry of the sources (photon scattering in the not-spherical geometries presents in the accretion disks, photon propagation in magnetized plasmas etc.);

- fundamental physics, related to quantum electrodynamics (photon propagation in strong magnetic fields), general relativity (photon propagation in strong gravitational fields) and quantum gravity and Lorentz-invariance violation.

The $\mathrm{X}$ ray polarisation in the $1 \mathrm{keV}: 100 \mathrm{keV}$ region can be measured by looking at the distribution of the direction of emission of a K-shell electron emitted in the photoelectric effect, since the distribution is $100 \%$ modulated if the incident radiation is $100 \%$ linearly polarised. The electron is preferentially emitted orthogonally to the incidence direction. The technical challenges in this type of measurement are the small photoelectron range (typically less than $1 \mathrm{~mm}$ in gas) and the multiple Coulomb scattering. One of the most promising detection system for the measurement of the X-ray polarisation is based on the use of a Micro Pattern Gas Detector, with a GEM and a finely pixelized readout anode, directly connected to a custom low noise ASIC, implementing on board amplification, shaping and self trigger functionalities (see Figure 1).

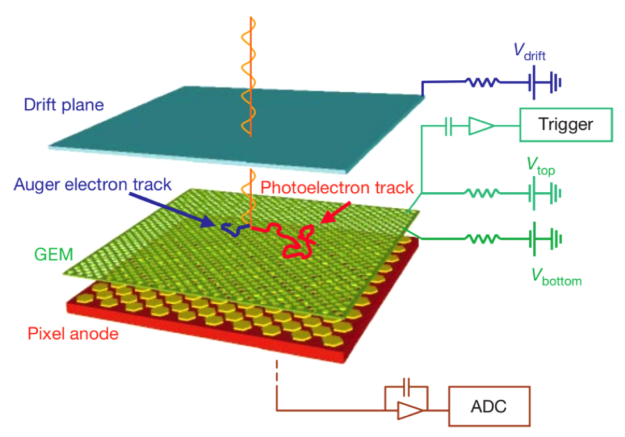

Figure 1: The basic principle used for the X-ray polarisation measurement proposed for the XIPE ESA mission [1]

- Anti-deuteron search

Anti-deuterons are produced by the co-annihilation and decay of weakly interacting massive particles. Several Dark Matter models, of disparate nature, predict a sizeable amount of antideuterons below few $\mathrm{GeV} / \mathrm{n}$, where the astrophysical background due to secondarily produced particles in the interstellar medium is strongly suppressed by the reaction kinematic. The search for low-energy cosmic anti-deuterons would hence offer a potential breakthrough in an unexplored phase space for DM. Among the indirect DM-detection techniques, the search of low energy anti-deuterons is the only unexplored observational channel, due to the very low flux and the large anti-proton background. The goal of the GAPS experiment [2] is to capture anti-deuterons in a target material and form an exotic atom in an excited state. The exotic atom would quickly decay, producing X-rays of characteristic energies and an additional pion signature from nuclear annihilation, which would allow to clearly distinguish anti-deuterons from anti-protons (see Figure 2). The GAPS detection concept has been proven with ground test at particle accelerator. An high-altitude balloon test was done in 
2012 with six pixellated $\mathrm{Si}(\mathrm{Li})$ detectors, for the first time operated in space. More details on the experiments can be found in reference [2].

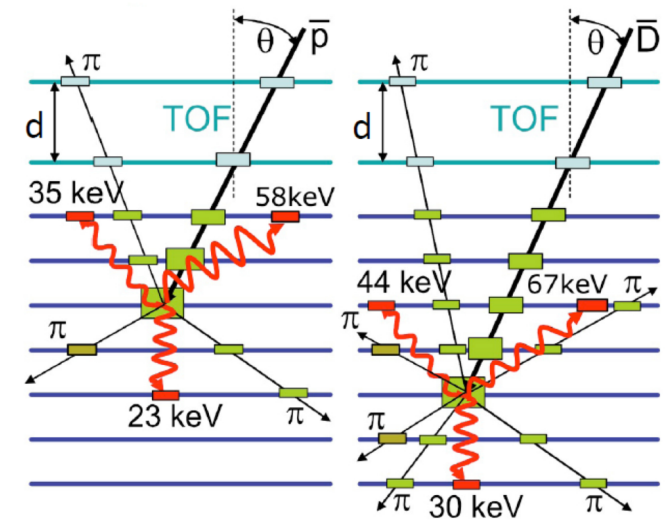

Figure 2: Basic GAPS detection concept

\section{Charged cosmic rays in the TeV-PeV energy region}

The experimental physics of charged cosmic rays by means of direct measurements has to face with two mean challenges: the measurement of energy spectra and composition of cosmic rays in the knee region $(\simeq 1 \mathrm{PeV})$ and the antiparticle spectra determination at high energy, well above 1 $\mathrm{TeV}$. The flux of cosmic rays, dominated by nuclei of galactic origin, covers a wide energy range which extends up to $10^{20} \mathrm{eV}$. Below $10^{15} \mathrm{eV}$ the spectrum decreases with energy approximately as a power law and the composition is roughly similar to that of ordinary matter. The most likely acceleration sites within the Galaxy are supernova remnants, where the shock wave generated by the exploding star propagates into the surrounding medium, typically for a few thousand years, accelerating particles to very high energies. After being released from the source regions, cosmic rays remain confined within the Galaxy for 10 millions of years, undergoing energy loss and nuclear interactions. At the knee energy region $\left(\simeq 10^{15} \mathrm{eV}\right)$ the inclusive spectrum becomes suddenly steeper and the composition progressively heavier. At these energies both the galactic accelerators and the confinement mechanism are believed to manifest their energy limits. Currently the knee region is accessible only indirectly, by detecting on ground the cosmic rays produced atmospheric showers. Only the spectra of groups of elements are measured and the results are considerably model dependent, for what concerns both the energy reconstruction and the elemental identification. Latest results on the light $\mathrm{CR}$ component $(\mathrm{p}+\mathrm{He}$ ) still show manifest incompatibilities (see Figure 3): the hybrid measurement by ARGO-YBJ and LHAASO [3] indicates a knee starting point around 0.6 $\mathrm{PeV}$, in disagreement with the results from the KASCADE array [4, 5] which has found a spectral break around 3-4 PeV. Direct CR detection allows unambiguous elemental identification, but suffers from severe limitations on the instruments exposure, due to the space location.

For what concern the positron component in cosmic rays, there is a strong evidence for an increase with energy of the positron fraction $\left(J\left(e^{+}\right) /\left[J\left(e^{+}\right)+J\left(e^{-}\right)\right]\right)$. The PAMELA, Fermi LAT and AMSO2 space based experiments together clearly show an excess between a few $\mathrm{GeV}$ and few 


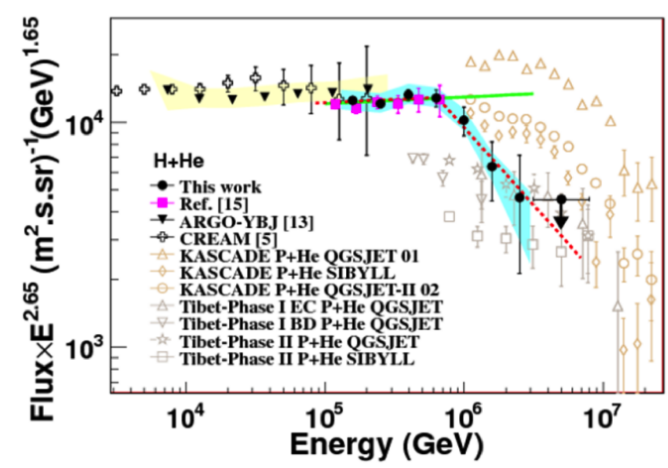

Figure 3: Collection of data from reference [3]. Markers labelled as "This work" refers to a combined analysis of ARGO-YBJ and LHAASO experiments.

hundred $\mathrm{GeV}$ compared to the secondary contribution due to the cosmic ray interaction in the interstellar medium. These observations call for additional sources of positrons, a candidate for which would be pulsars (or pulsars-wind nebulae) or else annihilation or decaying dark matter. PAMELA experiment has not detected any significant amount of antiproton flux beyond what expected from secondary interstellar medium production (see Figure 4). This fact puts strong constraints on any model of cosmic ray antiparticle production in the Galaxy. The present limit in the antiparticle
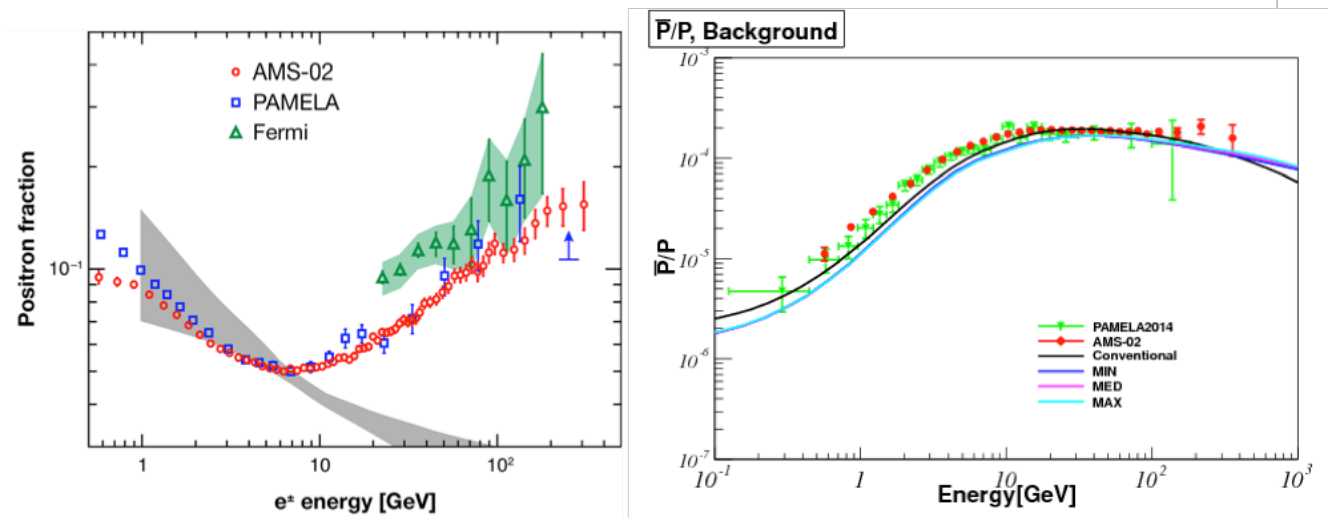

Figure 4: Latest measurements of the positron fraction (left plot) and the antiproton-to-proton ratio (right plot).

fluxes measurements for currently existing experiments are $500 \mathrm{GeV}$ for the positron component and $350 \mathrm{GeV}$ for the antiproton one. Being able to measure the antiparticles fluxes at higher energies, above the $\mathrm{TeV}$ region, would allow to distinguish between different models of production and in particular to understand whether the observed overabundance of positrons is due to the presence of dark matter in the Galaxy or is due to a some kind of astrophysical source. In addition to the limit of the acceptance, the antiparticle measurements are made difficult by the requirement of the charge sign identification, that requires the use of a magnetic spectrometer. The challenge for a new generation experiment in direct cosmic ray detection is to measure both the knee region and 
the antimatter component above $\mathrm{TeV}$ energies. One possibility is to combine two innovative techniques in a single experiment: a very large acceptance calorimeter (several $\mathrm{m}^{2} \mathrm{sr}$ ) and an extremely performing spectrometer with a Maximum Detectible Rigidity (MDR) larger then $10 \mathrm{TV} / \mathrm{c}$. Today this is made possible by exploiting and joining the ideas that form the basis of two innovative projects: CaloCube [6] and SR2S [7].

- An interesting innovative approach to calorimetry in space is the one followed by the CaloCube R\&D project, which has studied the possibility to directly detect high-energy cosmic rays by means of an isotropic and homogeneous calorimeter detecting particles from nearly all the incoming directions. The early proposed design of CaloCube is a 3D mesh of small cubic scintillating crystals, read-out by two photo-diodes each. The cubes are separated by a small gap that has two purposes: to increase the overall dimensions of the device (thus increasing the acceptance) and to accommodate support structures and sensors. Choosing as scintillating material the Thallium-doped Cesium Iodide as compromise to provide good performances for detection of both nuclei and e.m. particles, and assuming $2000 \mathrm{~kg}$ of total mass, the estimated performance is an energy resolution of 35-45\% (RMS) for protons with an effective geometric factor of $3-4 \mathrm{~m}^{2} \mathrm{sr}$.

- The Space Radiation Superconducting Shield (SR2S) European project aims at studying a large superconducting toroid magnet to protect the human habitat from the ionising radiations coming from galactic cosmic ray during long term missions in deep space. $\mathrm{The}_{\mathrm{MgB}}$ superconducting material is assumed to be used in order to produce a high intensity magnetic field all around the habitat module. A possible schematic design is shown in Figure 5.

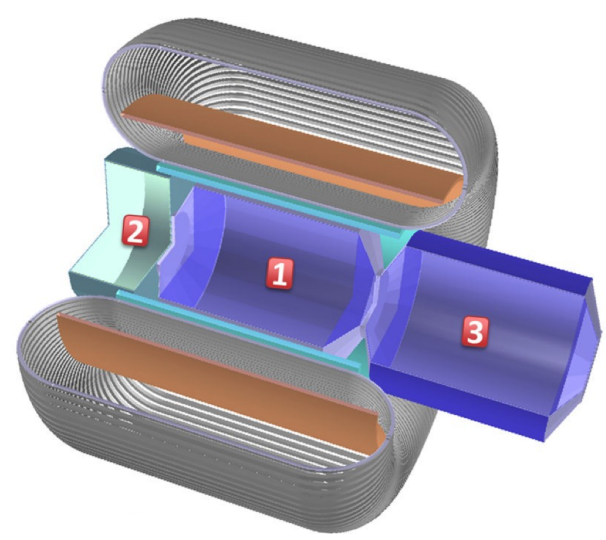

Figure 5: A possible schematic design of a Space Radiation Superconducting Shield. 1 is the Columbus module, 2 the fuel tank and 3 an additional service module

The $\mathrm{MgB}_{2}$ material is now widely used as a superconductor at a low temperature (as well as $\mathrm{NbTi}$ and $\mathrm{NbSn}$ ), and has the characteristic parameters suitable for its use in superconducting magnets system in space. First, it can be operated at a temperature much higher than the other materials, up to $20 \mathrm{~K}$, with a high current density in high magnetic field. Second, $\mathrm{MgB}_{2}$ is thermodynamically more stable, hence guaranteeing a large safety margin against quenching. Third, it is possible to construct cables with relatively low density by means of a substrate 
made of aluminium, as those developed by Columbus $\mathrm{Srl}$ for space application, with a mean density of $3.4 \mathrm{~g} / \mathrm{cm}^{2}$.

A future innovative experiment for cosmic rays at high energy can take advantage of both the results of CaloCube and SR2S, by a proper combination and optimisation in a configuration suitable for the detection of particles in space.

In the following, a possible configuration for a future experiment is presented considering a total mass of $2000 \mathrm{~kg}$ for the calorimeter and $2000 \mathrm{~kg}$ for the material of the magnetic system. The overall geometry shape is cylindrical, with a cylindrical calorimeter surrounded by a toroidal magnetic system armed by six cylindrical layers of micro-strip silicon detector to form a magnetic spectrometer.

\subsection{The calorimeter}

For what concern the calorimeter, ideally it should be spherical in shape, thus having all the external surface available for particle detection. A cylinder with the same diameter and length could be anyway an adequate option. Moreover, the choice of the shape of the single detection unit (each crystals) and their mutual arrangement should allow a three-dimensional tessellation of the available volume, hence avoiding the presence of escape planes. In fact, due to the mass constraints, the total depth of the calorimeter in term of interaction lengths will never be very deep for a space experiment (no more than 2-3 $\lambda_{I}$ ), and the main energy deposit detected by the light sensors will come from the electromagnetic component of the shower (produced by decay of neutral pions). The typical width of the electromagnetic shower is quite narrow and the presence of escape planes would increase the event-by-event fluctuations in the total energy deposit, hence worsening the energy resolution. In order to fulfill this design requirements, a possible solution is to use basic crystals shaped as hexagonal prisms ( $3 \mathrm{~cm}$ typical size), as shown in Figure 6 (top left).

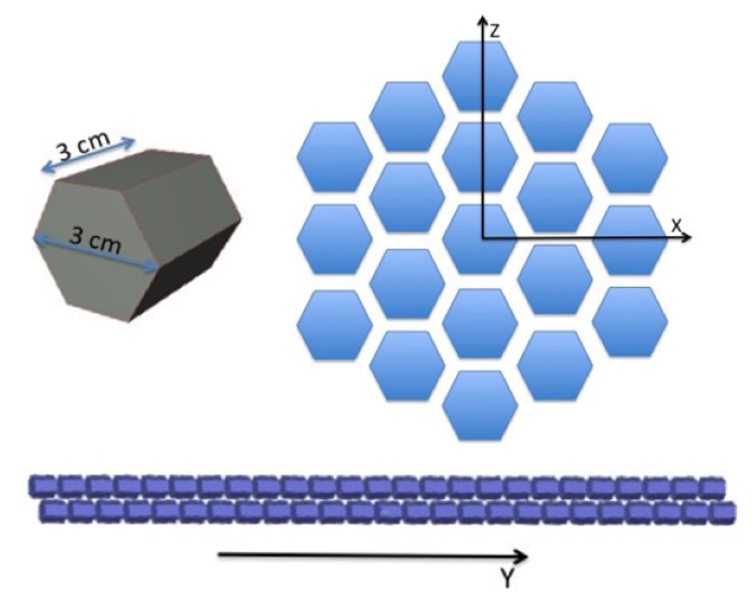

Figure 6: A possibile configuration for the cylindrical calorimeter

As an example, the disposition of 19 crystals on the plane X-Z is shown in Figure 6 (top right). Thanks to the hexagonal shape, a gap of $8 \mathrm{~mm}$ among crystals is possible without creating any significant escape plane. Along the $\mathrm{Y}$ axis (see Figure 6, bottom) 25 crystals are also arranged 
with a gap of $8 \mathrm{~mm}$ between each other, and two adjacent strings of crystals are displaced by half crystal length in a such way that in any direction no significant escape plane will be present. A possible solution for a global conceptual design of the cylindrical calorimeter is shown in Figure 7. It is made by 637 strings, each one composed by 25 crystals and arranged in such a way that the overall shape of the calorimeter is a cylinder with approximately the same dimensions in diameter and length. In the figure, each tube represents a string of crystals. In this configuration the total number of crystals is 15925 and the total Geometric Factor is $13.2 \mathrm{~m}^{2} \mathrm{sr}$. The analyses show that

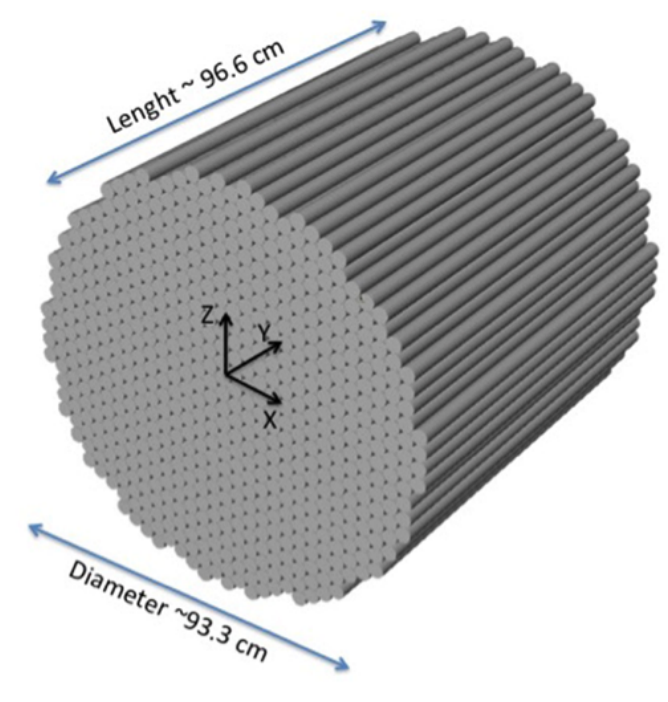

Figure 7: The global conceptual design of the cylindrical calorimeter

for cosmic ray nuclei measurements heavy scintillating material like BGO or LYSO are preferable with respect to lighter material such as CsI. The calorimeter shown in Figure 7 has a weight of 2000 $\mathrm{kg}$ in case of BGO crystals. The described calorimeter configuration has several advantage with respect to the original CaloCube design proposal. Thanks to the hexagonal shape of the crystals no escape planes are present; the gaps among crystals can be greater $(8 \mathrm{~mm})$ without significant loss of energy resolution thus increasing the total geometric factor; the BGO material is preferable for nuclei cosmic ray energy measurements. The increasing performance of this cylindrical calorimeter is shown in Figure 8 where the energy resolution for $1 \mathrm{TeV}$ protons as function of the effective geometric factor is plotted for the cylindrical and the cubic configurations, for the same weight (2000 kg), same scintillating material (BGO) and same gap size between crystals $(8 \mathrm{~mm})$. The effective geometric factor is defined as the overall geometric factor multiplied by the selection efficiency, which varies depending on the chosen selection criteria. The simulation demonstrate the excellent performance of this detector for cosmic ray nuclei measurements: an effective geometric factor approaching $7 \mathrm{~m}^{2} \mathrm{sr}$ and an energy resolution between $27 \%$ and $37 \%$ (RMS).

\subsection{The magnetic spectrometer}

To identify the charge sign of the particles and measuring also their rigidity, the calorimeter should be coupled to a magnetic spectrometer. The most appealing configuration is a toroidal magnetic system surrounding the calorimeter. The major reasons for this are the efficient use of 


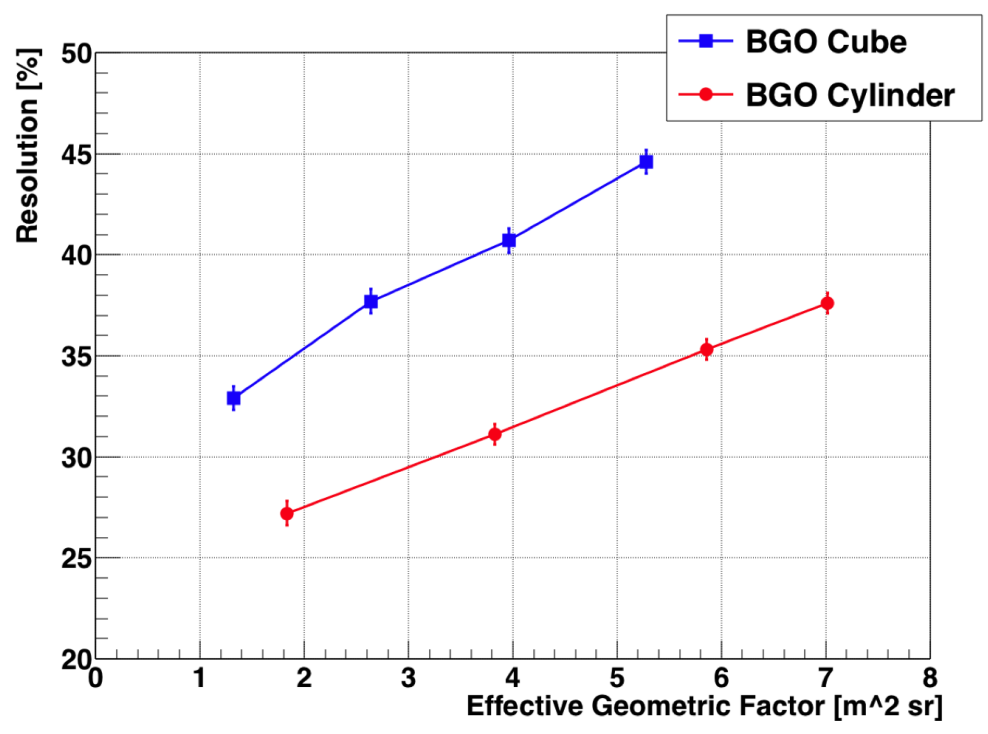

Figure 8: Comparison of the performances expected by a cubic and a cylindrical shaped $2000 \mathrm{~kg}$ BGO calorimeter

the produced magnetic field to bend the particle trajectories coming from the outer space and the null resulting magnetic moment of this kind of magnetic system. The main parameters (geometric dimensions, current and magnetic field) essentially depend on the used superconducting cable, on the operational temperature, on the total volume available as well as on the mass budget. They must be optimised taking into account the relationship between the maximum current density and the maximum magnetic field permitted by the $\mathrm{MgB}_{2}$ superconducting material. In the following a mass budget of $2000 \mathrm{~kg}$ is assumed as well as a cylindrical volume to install the experiment with a maximum diameter of $3.5 \mathrm{~m}$. The assumed working temperature is $10 \mathrm{~K}$. In Figure 9 a scheme of a magnetic system build with four coils is represented together with a possible disposition of the tracking system, composed by six cylindrical layers of silicon micro-strip detectors. The number of coils in the system can be chosen as a compromise between the requirements of a high MDR and a large effective geometric factor. By increasing the number of coils, we obtain a larger MDR (and a better uniformity of the field); however, the effective geometric factor is reduced due to the presence of the coils that shield the particle trajectories.

In Figure 10 six possible magnetic configurations are presented, with different number of coils (between two and twelve).

In order to evaluate the performances of the magnetic spectrometer an assumption on the position measurement resolution of the tracking system should be carried out. With the currently available technology for the silicon micro-strips detectors a reasonable value for the spatial resolution is $10 \mu \mathrm{m}$. In Figure 11 the performance of such a magnetic spectrometer in terms of MDR distribution is shown. To produce these plots a simulation has been done considering an isotropic flux of cosmic ray protons and the following selection criteria:

- only the upper two thirds of the total acceptance has been considered; 


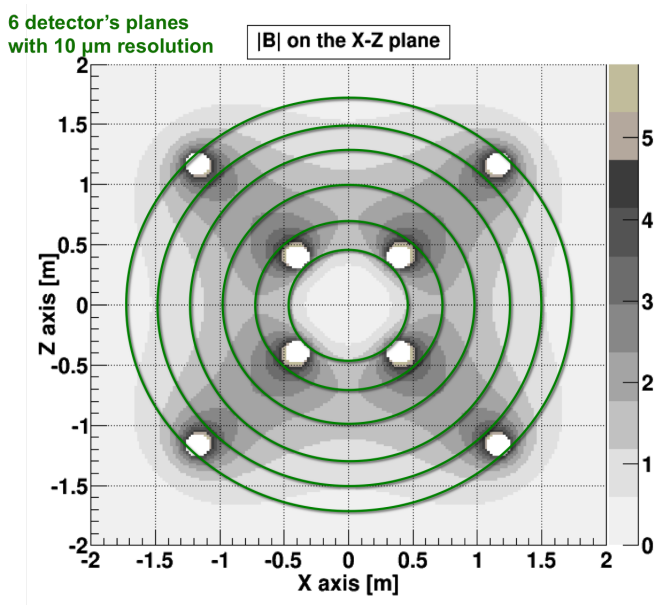

Figure 9: A possible scheme of a toroidal magnetic system, made with 4 coils, surrounding the cylindrical calorimeter. 6 layers of detectors are schematically shown. The magnetic field is reported in Tesla.
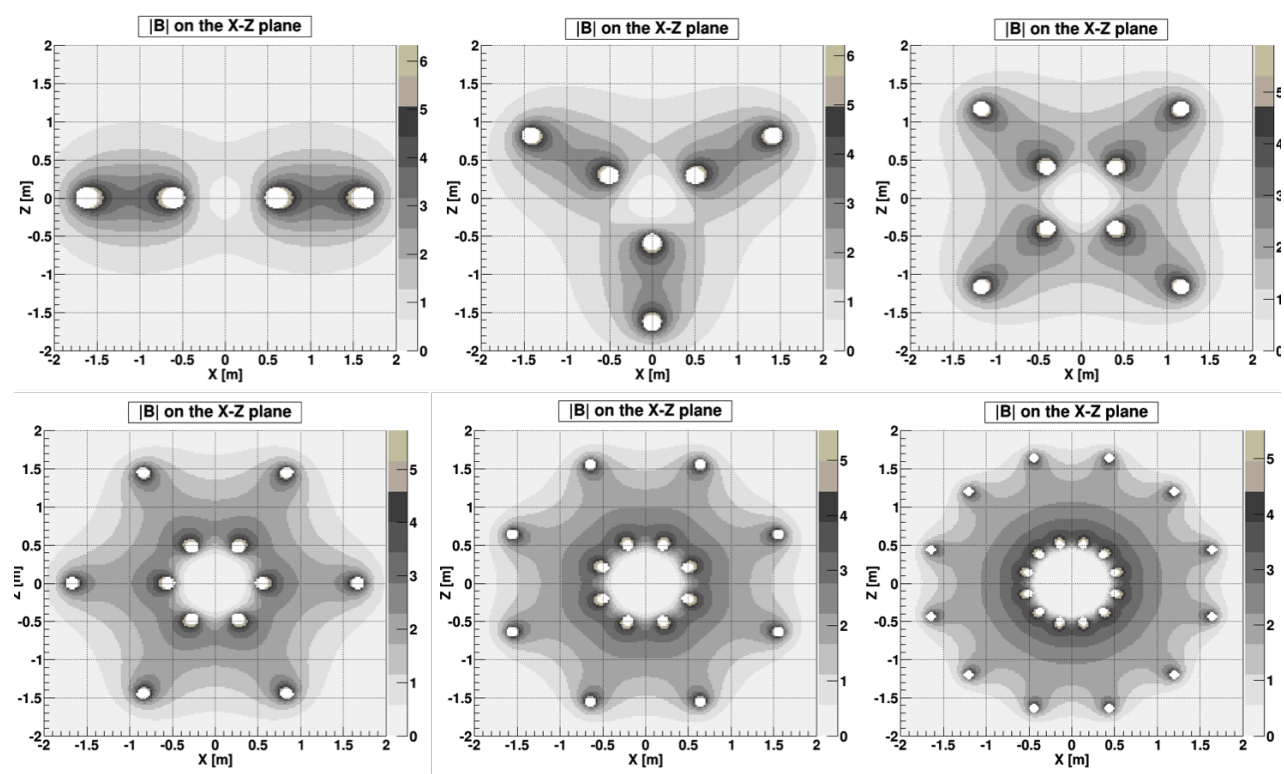

Figure 10: 6 possible different magnetic configurations, with different number of coils (from 2 up to 12). The magnetic field is reported in Tesla.

- the particle trajectories must cross all the six planes of the tracking system;

- the tracks must not pass through the magnetic coil material.

A Maximum Detectible Rigidity greater then $10 \mathrm{TV} / \mathrm{c}$ can be obtained, with an effective geometric factor of a few $\mathrm{m}^{2} \mathrm{sr}$. An experiment in space with these performances, collecting data for several years, should be able to measure antiproton and positron fluxes well above few TeV. 


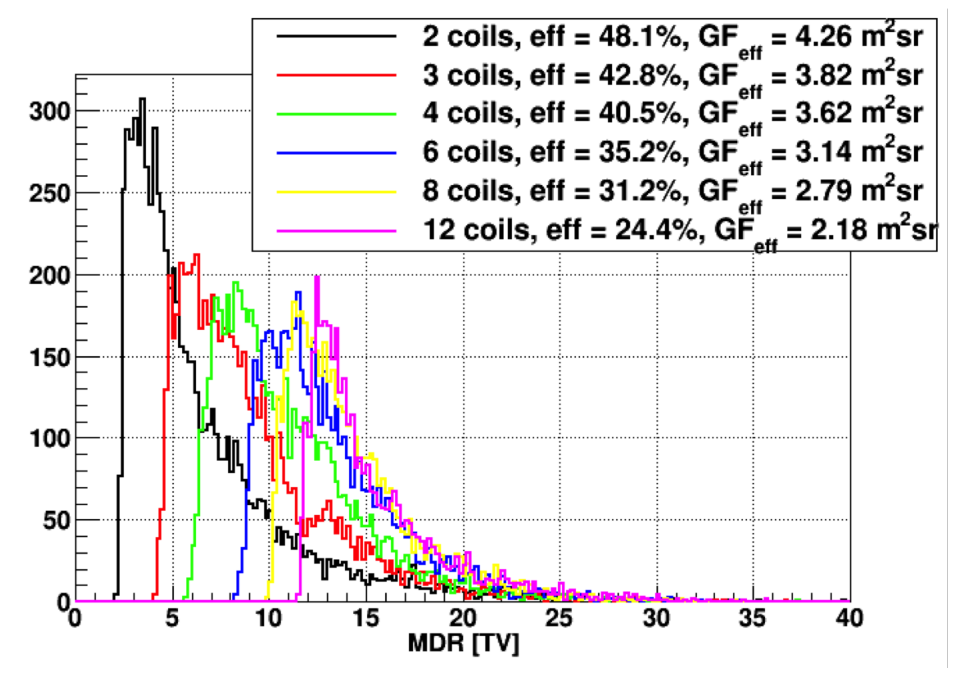

Figure 11: Maximum Detectable Rigidity distributions for the 6 different magnetic configurations

\section{References}

[1] P. Soffita et al., Exper.Astron. 36 (2013) 523-567

[2] T. Aramaki et al., Astro. Ph. 74 (2016) 6-13

[3] B. Bartoli et al., PRD 92 (2015) 092005

[4] T. Antoni et al., Astro. Ph. 24 (2005) 1-25

[5] W.D. Aple et al., Astro. Ph. 47 (2013) 54-66

[6] N. Mori et al., NIM A 732 (2013) 311-315

[7] B. Romain, Physics Procedia 67 (2015) 264- 269 